Aim of the study: Examination of esophagojejunal (EJ) anastomosis with aqueous contrast swallow after total gastrectomy is still routinely conducted by many centres. The present study aimed to answer the question: Is it necessary to evaluate EJ anastomosis in terms of leakage by having every patient drink oral contrast agent before initiation of oral food intake after total gastrectomy (TG) performed due to gastric cancer?

Material and methods: Clinical and radiological results of patients on whom total gastrectomy was performed due to gastric cancer between January 2013 and December 2016 were retrospectively reviewed. Diagnostic method used for patients in whom leak developed and therapeutic interventions were assessed. Evaluation results from aqueous contrast agent and clinical, laboratory, and tomographic findings were studied. Results: Sixty of the 69 patients who underwent total gastrectomy with a diagnosis of gastric adenocarcinoma swallowed aqueous contrast agent on postoperative day $7 \pm 2$ days and were evaluated in terms of anastomotic leak. Leak developed in $14 \mathrm{pa}$ tients (20.2\%), 10 of whom ingested contrast agent. Leak was identified in 6 of those patients; however, diagnosis was made with multislice computed tomography (CT) in four patients (40\%). The sensitivity of the examination with aqueous contrast agent was $60 \%$

Conclusions: Evaluating anastomotic leak with aqueous contrast agent after TG has low sensitivity, and it would be wise to resort to this procedure in cases with clinical suspicion, rather than routinely performing it in every patient.

Key words: anastomotic leak, diagno stic imaging, esophagojejunal anastomosis, gastrectomy.

Contemp Oncol (Pozn) 2017; 21 (3): 224-227 DOI:https://doi.org/10.5114/wo.2017.70112

\section{Examination of anastomotic leak with aqueous contrast swallow after total gastrectomy: Should it be carried out routinely?}

\author{
Ulaş Aday, Ebubekir Gündeş, Hüseyin Çiyiltepe, Durmuş A. Çetin, \\ Selçuk Gülmez, Aziz S. Senger, Kamuran C. Değer, Mustafa Duman
}

Department of Gastroenterological Surgery, Kartal Kosuyolu High Speciality Education and Training Hospital, ístanbul,Turkey

\section{Introduction}

Esophagojejunal (EJ) anastomotic leak that develops after total gastrectomy (TG) is an important complication leading to an increase in morbidity and mortality. Postoperative anastomotic leak develops at a rate of $1 \%$ to $20 \%$, and is associated with high mortality [1-5]. Symptomatic leaks are usually identified within the first 10 days, and result in death at a rate of 25\% to 50\% [4]. Examination of EJ anastomosis with aqueous contrast swallow (ACS) before the initiation of oral food intake after TG is still carried out by many centres [5]. However, some studies do not recommend routine ACS use because its sensitivity is low $[1,2,4,5]$.

In our clinic, oral intake is initiated in patients who undergo TG due to gastric malignancy after anastomotic integrity is examined with ACS. This study aimed to find an answer to the question: Is it necessary to examine EJ anastomosis with ACS in every patient?

\section{Material and methods}

Data related to 134 patients who underwent resection for curative purposes due to gastric cancer between January 2013 and December 2016 were retrospectively reviewed. Cases diagnosed with adenocarcinoma by endoscopic biopsy were included in the study; patients who underwent surgery due to lymphoma, stromal tumour, neuroendocrine tumour, or haemorrhage were excluded. Two patients underwent proximal gastrectomy, 61 underwent subtotal gastrectomy, and 71 underwent TG. Of the 71 TG patients, the data, archive files, electronic archive data of the hospital, and records of radiological images of 69 patients were obtained by retrospective review. This study was approved by the institutional review board of Kartal Dr. Lütfi Kırdar Education and Research Hospital (prot: 2016/514/86/3).

Total gastrectomy was performed with D2 lymphadenectomy by dividing the jejunum at the $20^{\text {th }} \mathrm{cm}$ from the ligament of Treitz and was attached marginally-laterally to the distal oesophagus with a circular stapler. Jejuno-jejunal anastomosis was performed by hand to ensure that it would be marginal lateral to approximately $40 \mathrm{~cm}$ distal of EJ anastomosis. Duodenal and jejunal stumps were closed with a linear stapler. Un-bloc resection was conducted in cases with adjacent organ invasion and those that were compatible with $\mathrm{RO}$ resection. Two soft drains were used in order to see the duodenal stump and EJ anastomosis line. All patients had nasojejunal feeding tube inserted intraoperatively, and low-dose enteral feeding was initiated on the postoperative first day. Enteral feeding was gradually increased to the necessary dosage in patients with good tolerance. Total parenteral nutrition was provided for patients who could not tolerate enteral feeding by tube. 
All patients whose general condition was sufficient for them to swallow contrast agent and to be transferred to a radiology department were asked to ingest 100 cc of pure Ultravist $300{ }^{\circledR}$ (Bayer Schering Pharma, Mijdrecht, the Netherlands), on postoperative day $7 \pm 2$ days, and EJ anastomotic leak was examined with anterior-posterior and lateral-oblique images taken using fluoroscopy. Soft food intake was started in patients who were asymptomatic and in whom anastomotic leak was not detected; normal food intake was achieved gradually. In the event of leak detection in the ACS examination, food intake was started in asymptomatic patients after a delay. In patients in whom leakage and accompanying clinical symptoms were detected, fluid collection or abscess was investigated using tomography. In the presence of clinical leak suspicion for patients who did not have leak indicated in ACS evaluation, tomographic examination was conducted by having the patients take oral and intravenous contrast agent. Radiological leak was defined as extravasation from the lumen after ACS intake monitored with fluoroscopy, observation of contrast agent outside the lumen on multislice computed tomography (CT), detecting an abscess adjacent to the anastomosis, or finding a defect in the anastomosis line. Also, the diagnosis of anastomotic defect with gastroduodenoscopy was defined as leakage. The definition of clinical leak was specified as the presence of intestinal or purulent content from the surgical incision or drains, fever, abdominal pain, C-reactive protein (CRP) or leucocyte count increase, or detection of leakage on re-laparotomy for abdominal sepsis $[1,2,3,7]$. The highest CRP value on postoperative day 3 or 4 was recorded based on patient postoperative laboratory data. Hospital stay was defined as the time elapsed from the day of surgery to discharge and death occurring during hospitalisation or within the first 30 days after surgery was classified as hospital mortality.

\section{Statistical analysis}

SPSS software (version 21.0; SPSS Inc., Chicago, IL, USA) was used for statistical analysis of the data. Continuous variables were evaluated using Kolmogorov- Smirnov normality test. Mean value and minimum-maximum values of continuous variables were recorded. Mann-Whitney $U$ test was used for intergroup comparisons. Categorical groups were compared with chi-square test. $P<0.05$ was accepted as statistically significant.

\section{Results}

Seventy-one patients were diagnosed with adenocarcinoma and had D2 lymph node dissection and TG performed. Two patients were excluded from the study because data could not be obtained. The mean age of 69 patients included was 61.7 years (range: 32-91 years); the female:male ratio was 1 : 4.2. Along with gastrectomy, splenectomy was performed on nine patients, distal pancreatectomy on four, cholecystectomy on three, transverse colon resection on one, resection of liver segments 2 and 3 on one patient, and left adrenal gland resection on one patient. Leak developed in a total of 14 patients (20.2\%).
The mean age of the patients in whom leak developed was 69 years (range: 53-78 years), and mean age of the patients in whom leak did not develop was 62 years (range: 30-91 years), which was found to be statistically significant $(p=0.048)$. The mean highest CRP value measured on day 3 or 4 was $19.95 \mathrm{mg} / \mathrm{dl}$ (range $8.8-35 \mathrm{mg} / \mathrm{dl}$ ) in patients with leak; the same value was $10.4 \mathrm{mg} / \mathrm{dl}$ (range: 2.4-70 mg/dl) in the group that did not develop any leaks, which was also statistically significant $(p<0.001)$. Mean length of hospital stay was 11 days (range: 8-48 days) in the group that did not develop leak, and 30 days (range: 3-140 days) in the group that developed leak, which was also determined to be statistically significant $(p<0.001)$. A total of three patients (4.3\%) died from sepsis associated with leak. The results for both groups are summarised in Table 1.

Sixty patients were examined with ACS for EJ anastomosis, but this was not possible in nine patients (Fig. 1). Leak developed in four of the nine patients who could not be evaluated with ACS. Clinical diagnosis of leak was made with re-laparotomy conducted due to abdominal sepsis that developed before the postoperative seventh day in two patients, by endoscopy for one patient, and by having seen intestinal content in abdominal and thoracic drains in one

Table 1. Characteristics of patients with and without anastomotic leak

$\begin{array}{lccc} & \begin{array}{c}\text { No Leak } \\ (n=55)\end{array} & \begin{array}{c}\text { Leak } \\ (n=14)\end{array} & P \text { value } \\ \text { Age (years) } & 62(30-91) & 69(53-78) & 0.048^{*} \\ \text { Gender } & & & \\ \text { Female } & 18(26 \%) & 3(4.3 \%) & 0.412 \\ \text { Male } & 37(53.6 \%) & 11(15.9 \%) & \\ \text { CRP (mg/dl) } & 10.4(2.4-70) & 19.9(8.8-35) & <0.001^{*} \\ \text { LOS (days) } & 11(8-48) & 30(3-140) & <0.001^{*} \\ \text { CRP-C-reactive protein; } \text { LOS - length of hospital stay; }{ }^{*} p<0.05 & \end{array}$

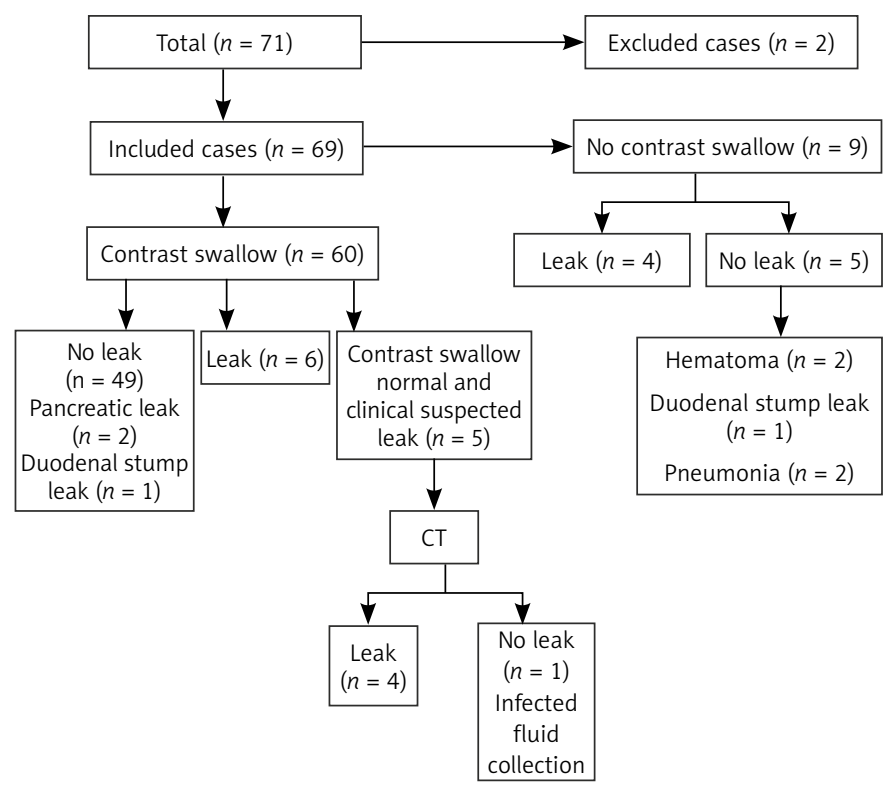

Fig. 1. Flow chart of this study 
Table 2. Clinical features of patients who developed leak and were examined by ACS

\begin{tabular}{|c|c|c|c|c|c|c|c|}
\hline $\begin{array}{c}\text { Patient } \\
\text { no. }\end{array}$ & Fever & $\begin{array}{c}\text { Abdominal } \\
\text { pain }\end{array}$ & Tachycardia & $\begin{array}{l}\text { Suspicious Drain } \\
\text { content }\end{array}$ & $\begin{array}{c}\text { CRP level on day } \\
3 \text { or } 4\end{array}$ & $\begin{array}{c}\text { Diagnosis with } \\
\text { ACS }\end{array}$ & $\begin{array}{l}\text { Treatment } \\
\text { method }\end{array}$ \\
\hline 1 & - & - & + & - & 8.8 & + & Conservative \\
\hline 2 & + & + & + & - & 24.1 & + & Interventiona \\
\hline 3 & + & - & + & + & 16.2 & + & Interventional \\
\hline 4 & - & + & + & - & 17 & + & Surgical \\
\hline 5 & + & + & - & + & 22.6 & + & Interventional \\
\hline 6 & + & + & + & - & 20.8 & + & Conservative \\
\hline 7 & + & + & + & + & 35 & - & Interventional \\
\hline 8 & + & + & + & + & 13.7 & - & Interventiona \\
\hline 9 & + & + & + & + & 18.5 & - & Conservative \\
\hline 10 & - & + & + & + & 25.9 & - & Conservative \\
\hline
\end{tabular}

Table 3. Specificity, sensitivity, positive predictive value, and negative predictive value of routine aqueous contrast swallow examinations in detecting EJ anastomotic leakage (\%)

$\begin{array}{cc}\text { Specificity } & 100 \\ \text { Sensitivity } & 60 \\ \text { PPV } & 100 \\ \text { NPV } & 92.5 \\ \text { NPV - negative predictive value; PPV - positive predictive value }\end{array}$

patient. In the other five patients who could not be examined with ACS, leak was investigated in EJ anastomosis by performing multislice CT before the postoperative seventh day due to clinical suspicion. Haematoma was detected in two patients, duodenal stump leak in one, and pneumonia in the remaining two patients (Fig. 1). Leak was present in 10 of the 60 patients examined with ACS; however, leak diagnosis was made by ACS in only six patients $(6 / 10$, $60 \%$ ). Leak was identified by CT carried out due to ongoing clinical suspicion in an additional four patients. All patients with leak diagnosis with ACS were given tomography to plan treatment. A thoracic tube was inserted due to fluid accumulated in the left hemithorax in 2 of the 6 patients diagnosed by ACS; percutaneous drainage was performed in one patient due to intraabdominal abscess. Colostomy was performed on another one of these six patients on postoperative day 9 due to transverse colon fistula in which a 5-mm defect was detected in the EJ anastomosis and closed with suture. No other invasive procedure was performed on the other two patients. Oral food intake was initiated for 49 of 60 patients whose EJ anastomosis was examined by ACS without any sign of leak. Food intake was delayed in 3 patients because of pancreatic and duodenal stump leak (Fig. 1). Abdominal pain, tachycardia, suspicious drain content and CRP elevation were detected in all of the 4 patients that had leak not identified by ACS. High fever ongoing for more than three days was present in three of these patients. It was determined that simultaneous wound site infection had developed in one of these patients and empyema had developed in another. Two patients who had leak but whose ACS examination was negative were treat- ed conservatively, and 2 patients were treated with percutaneous drainage (Table 2). While leak was identified in six patients with ACS, EJ anastomotic leak in four patients with negative ACS was identified with CT due to ongoing clinical suspicion. In ACS examination, sensitivity was established at $60 \%$, specificity at $100 \%$, and negative predictive value was $92.5 \%$ (Table 3 ).

\section{Discussion}

Incidence of EJ anastomotic leak in patients on whom TG is performed due to gastric cancer is reported in various ratios, from $1 \%$ to $20 \%$, in the literature [1-5]. Due to the fact that it results in high morbidity and mortality, early identification and therapeutic interventions are of great importance [3-6]. EJ anastomotic leak can occur after left gastric artery ligation because of decreased blood supply of the lower oesophagus. Lack of serosa in the oesophagus, the hiatal area of the anastomosis, represents a challenging location for surgery, and due to hypoxia related to hypoventilation in the lungs because of postoperative pain are other reasons of EJ anastomotic leak [1, 8].

Studies have reported that ACS examinations have low sensitivity, and it has also been stated that many centres still use this method $[1,9,10]$. Lamb et al. emphasised in a prospective study that the sensitivity of ACS examination is $66.6 \%$ and that it does not contribute to the examination of EJ anastomosis after TG [1]. In the present study, of 10 patients with leak, who swallowed contrast agent, leak was detected in 6 (60\%) and not found in 4 (40\%). Diagnosis of these four patients was made by tomography taken due to ongoing clinical suspicion. ACS examination could not be done for nine patients (13\%). Two of these patients underwent re-laparotomy due to abdominal sepsis. In addition to copious bilious content from drains, massive mediastinal fluid accumulation was observed in one of these patients on postoperative day 3. The patient, whose thoracic tube was filled with intestinal content, was lost on the same day. In the other patient, diagnosis was made endoscopically due to prolonged intensive care follow-up, and a therapeutic stent was inserted. Clinical findings such as fever, abdominal pain, purulent or intesti- 
nal content coming from drain, or CRP elevation are seen in most patients who develop anastomotic leak. Therefore, it is indicated that, rather than examining EJ anastomosis routinely with ACS, it should be carried out in the event of clinical suspicion $[2,5,11]$. Parameters such as fever, abdominal pain, suspicious content from drain, tachycardia, or CRP elevation were present in all of our patients with leak. Dutta et al. emphasised that CRP value over $18 \mathrm{ng} / \mathrm{dl}$ on postoperative days 3 and 4 has high sensitivity for anastomotic leak [11]. In our study, CRP values taken on postoperative day 3 or 4 were high in all patients with leak, and it was over $18 \mathrm{mg} / \mathrm{dl}$ in 10 of 14 patients (71.4\%) with leak.

Tomographic examination has some advantages. The rate of true diagnosis by tomography immediately after oral contrast intake is quite high. It is authoritative for identification of thoracic and abdominal abscesses, fluid collection, or haematoma and for choosing interventional procedures [4]. Aspiration risk is always present in ACS examination or barium swallow, and CT is a procedure that is more comfortably tolerated by patients $[9,10]$. In a study by Lee et al., it was stated that in cases with clinical suspicion of leak, fluoroscopy performed after a CT result of "probable leak" confirmed the leak definitively. Therefore, they emphasised use of fluoroscopy first because CT can give false positive results in cases with clinical suspicion of leak. They stated that fluoroscopic examination has advantages such as providing information on anastomotic stricture or presence of obstruction [7]. A study conducted by Hogan et al. yielded different results. Eight leaks were identified on CT and fluoroscopy in a total of 38 patients. Leak was suggested by ACS in three asymptomatic patients, but was confirmed as not present with CT and endoscopy. Therefore, they noted that false positive results could be obtained with ACS and the clinician could be misled [4]. In the present study, the leakage rate of anastomosis was higher than in the literature (20.2\%). Clinical progress was asymptomatic and oral intake was delayed in one patient in whom leak was detected by ACS (Table 2, Patient no. 1). However, leak was not confirmed with CT, and a false positive result may have been obtained with ACS. In our study, tomographic examination was superior to fluoroscopic examination performed with ACS because diagnosis of leak was made by $C T$ in four patients with clinical suspicion of leak despite negative ACS result. CT provides evaluation of abscess, fluid collection, and additional organ pathologies (pneumonia, haematoma, duodenal stump leak, etc.) that are not evaluated with fluoroscopy, and furthermore, $\mathrm{CT}$ directs treatment strategies. The limitations of this study include its retrospective nature, small number of cases, not having evaluations with CT for all patients in the study group, and not having had the opportunity to conduct a one-to-one comparison of studies aimed at diagnosis.

In conclusion, the authors are of the opinion that examining EJ anastomosis with ACS after TG need not be routinely employed due to low sensitivity. CT can be used primarily in the event of suspicion of leak with close clinical observation and monitoring of laboratory parameters. We think that tomographic evaluation with oral contrast is superior because of the advantages such as diagnosis of anastomotic leakage, identification of additional accompanying pathologies, and planning therapeutic interventions. After this study was conducted, routine examination of EJ anastomosis with ACS was discontinued at our clinic.

This retrospective study was approved by the institutional review board of Kartal Dr. Lütfi.

Kurdar Education and Research Hospital (prot: 2016/514/86/3).

The authors declare no conflict of interest.

\section{References}

1. Lamb PJ, Griffin SM, Chandrashekar MV, Richardson DL, Karat D, Hayes N. Prospective study of routine contrast radiology after total gastrectomy. Br J Surg 2004; 91: 1015-19.

2. Tonouchi H, Mohri Y, Tanaka K, Ohi M, Kobayashi M, Yamakado K, Kusunoki M. Diagnostic sensitivity of contrast swallow for leakage after gastric resection. World J Surg 2007; 31: 128-31.

3. Lee S, Ahn JY, Jung HY, et al. Clinical outcomes of endoscopic and surgical management for postoperative upper gastrointestinal leakage. Surg Endosc 2013; 27: 4232-40.

4. Hogan BA, Winter DC, Broe D, Broe P, Lee MJ. Prospective trial comparing contrast swallow, computed tomography and endoscopy to identify anastomotic leak following oesophagogastric surgery. Surg Endosc 2008; 22: 767-71.

5. Igor J, Poluksht N, Siegelmann-Danieli N, et al. The role of upper gastrointestinal swallow study in patients undergoing proximal or total gastrectomy. Isr Med Assoc J 2010; 12: 560-62.

6. Bruce J, Krukowski ZH, Al-Khairy G, Russell EM, Park KG. Systematic review of the definition and measurement of anastomotic leak after gastrointestinal surgery. Br J Surg 2001; 88: 1157-68.

7. Lee S, Ahn JY, Jung HY, et al. Clinical Outcomes of Postoperative Upper Gastrointestinal Leakage According to Treatment Modality. Dig Dis Sci 2016; 61: 523-32.

8. Deguchi Y, Fukagawa T, Morita S, Ohashi M, Saka M, Katai H. Identification of risk factors for esophagojejunal anastomotic leakage after gastric surgery World I Surg 2012; 36: 1617-22.

9. Upponi S, Ganeshan A, D'Costa H, Betts M, Maynard N, Bungay H, Slater A. Radiological detection of post-oesophagectomy anastomotic leak - a comparison between multidetector CT and fluoroscopy. Br J Radiol 2008; 81: 545-48.

10. Boone J, Rinkes IB, van Leeuwen M, van Hillegersberg R. Diagnostic value of routine aqueous contrast swallow examination after oesophagectomy for detecting leakage of the cervical oesophagogastric anastomosis. ANZ J Surg 2008; 78: 784-90.

11. Dutta S, Fullarton GM, Forshaw MJ, Horgan PG, McMillan DC. Persistent elevation of C-reactive protein following esophagogastric cancer resection as a predictor of postoperative surgical site infectious complications. World J Surg 2011; 35: 1017-25.

\section{Address for correspondence}

\section{Ulaş Aday}

Kartal Kosuyolu High Speciality Education and Training Hospital Gastroenterology Surgery Cevizli M

34685 Istanbul, Turkey

e-mail: ulasaday@gmail.com

Submitted: 26.05 .2017

Accepted: $\quad 25.06 .2017$ 